\title{
Fabrication of All-printed Organic TFT A rray on Flexible Substrate
}

\section{K oei Suzuki, K eiichiroh Yutani, M akito Nakashima, A tsushi O nodera, Satoshi Mizukami, Masaki K ato, Takanori Tano and Hidenori Tomono}

\author{
Ricoh Co., Ltd. \\ 16-1 Shinei-cho, Tsuzuki-ku, Yokohama, Kanagawa 224-0035, J apan
}

\begin{abstract}
The surface energy controlled ink-jet printing with ultraviolet (UV) irradiation on a novel polyimide film was developed for high-resolution electrode patterning. Minimum linewidth of electrode was $15 \mu \mathrm{m}$ and minimum space between two electrodes was $2 \mu \mathrm{m}$ respectively. $160 \mathrm{ppi}$ and $200 \mathrm{ppi}$ all-printed organic thin film transistor (OTFT) backplanes were fabricated by the surface energy controlled ink-jet printing for electrodes, conventional ink-jet printing for organic semiconductor (OSC) and several printing methods on plastic substrate. We have also fabricated a 300 ppi all-printed OTFT array on plastic substrate. Flexible electrophoretic displays (EPDs) driven by 200 ppi all-printed OTFT backplanes were also successfully demonstrated.
\end{abstract}

K eyword: printed electronics, ink-jet printing, surface energy controlled ink-jet printing, organic semiconductor

\section{Introduction}

Printed electronics are gaining attention as a technology that enables various electric devices such as organic thin film transistors (OTFTs) [1, 2, 3], RF-ID tags [4] and sensors [5] to be fabricated using printing process. Printing method has potential of low cost, low environmental impact and large area fabrication. Low cost and low environmental impact are because of small number of process steps, small amount of materials and high through-put. However, there are some issue to overcome such as resolution, electric property, process integration, and reliability to fabricate electronic devices by printing on plastic substrates.

For resolution, typical resolution of conventional ink-jet printing is around $50 \mu \mathrm{m}$. Therefore various methods have been proposed to improve resolution of printing using bank structures [2] and self-assembled monolayer [6] for OTFT applications. For resolution and process integration, there have recently been some aggressive reports of OTFT backplane for flexible electronic paper, 76 ppi [7] , 150 ppi [8] all-printed one using an off-set-based high-resolution printing, 150 ppi one using photo-assisted surface modification technique with screen-printing [9], 200 ppi all-printed one using microcontact printing [10]. Moreover, for electric property, OTFTs with high mobility of $0.4 \mathrm{~cm}^{2} / \mathrm{V} \mathrm{s}$ has been reported [11].

In previous work we developed the surface energy controlled ink-jet printing for electrode with a water based ink of poly (3, 4-ethylenedioxythiophene) poly (styrenesulfonate) (PEDOT/PSS) [12]. Here using Ag nanoparticles ink it was shown that minimum linewidth of electrode was $15 \mu \mathrm{m}$ and minimum space between two electrodes was 2 $\mu \mathrm{m}$. We successfully fabricated several resolution of all-printed OTFT backplane such as $160 \mathrm{ppi}$ [13] and $200 \mathrm{ppi}$ [14] on plastic substrate to integrate the process of several printing methods. Flexible 
electrophoretic displays (EPDs) driven by these all-printed OTFT backplanes were also successfully demonstrated. To show the scaling capability of our printing process, we have also fabricated a 300 ppi all-printed OTFT array without pixel electrodes on plastic substrate [15].

\section{Experimental}

\subsection{TFT structure and process}

Figure 1 shows a schematic cross-section of the all-printed OTFT backplane with bottom-gate bottom-contact structure on plastic substrate. The gate electrodes, the storage capacitor electrodes, the source/drain (S/D) electrodes and these lines were fabricated using Ag nanoparticles ink by the surface energy controlled ink-jet printing. The gate insulator was a novel polyimide film fabricated by spin coating. Small-molecule OSC for 200 ppi OTFTs and polymer OSC for 160 ppi OTFTs were fabricated by ink-jet printing under ambient conditions respectively. A fter the formation of OSC layer, the insulator and pixel electrodes were fabricated by screen printing. All of these layers were fabricated by several printing processes under ambient conditions. Maximum process temperature was $180^{\circ} \mathrm{C}$.

Organic Semiconductor

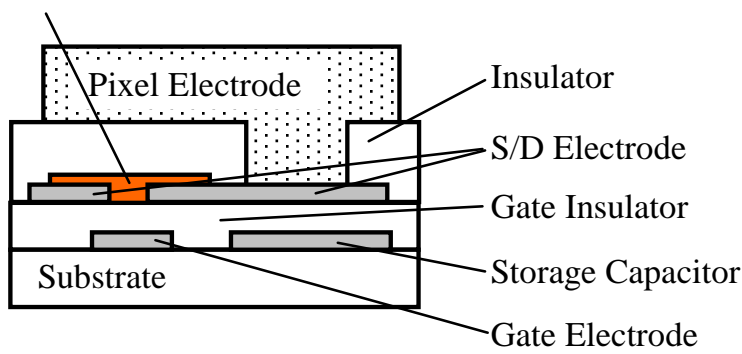

Figure 1. Cross-section of the all-printed OTFT backplane

To achieve all-printed OTFTs we choose the several printing methods. The surface energy controlled If printing was used for achieving the small surface roughness, the fine electrode patterning, and the precise controlled channel length. The conventional I) printing was used for the contamination-free printing because of non-contact printing without printing plate. The screen printing was used for the thick film formation.
2.2. Surface energy controlled ink-jet printing process

We have developed the surface energy controlled ink-jet printing with ultraviolet (UV) irradiation on a novel polyimide film for high-resolution electrode patterning (see Figure 2). The novel polyimide film was fabricated on the plastic substrate by spin coating, whose surface had low surface energy after post-baking in $\mathrm{N}_{2}$ condition at $180^{\circ} \mathrm{C}$ (Figure $2(\mathrm{a})$ )

(a)

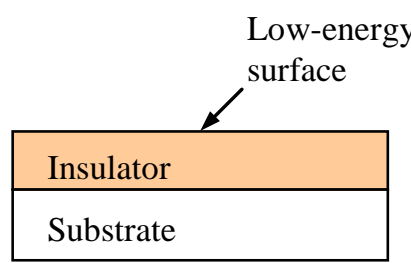

(b)
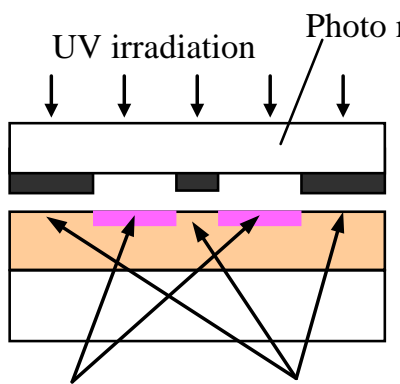

High surface

Low surface energy area energy area

(c)

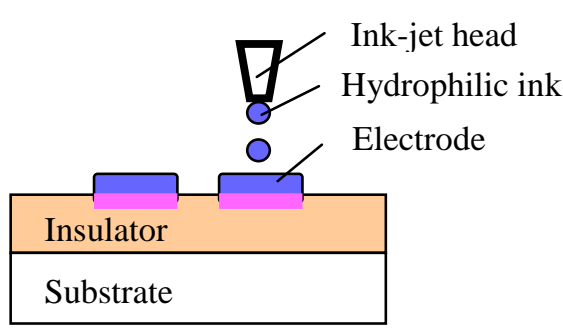

Figure 2. Schematic of the surface energy controlled ink-jet printing process (a) Fabrication of novel polyimide film, (b) Formation of areas with different surface energy by UV irradiation, (c) Fabrication of electrodes by ink-jet printing

After UV irradiation from super-high pressure mercury lamp through a photo mask from the front side of the substrate, the high surface energy area corresponding to electrode patterns and the low surface energy area were formed on the novel polyimide film surface (Figure 2(b)). Hydrophilic Ag nanoparticles ink was ink-jetted onto the high surface energy area and spread over the edge of the area (Figure 2(c)). After post-baking 
under ambient condition at $180^{\circ} \mathrm{C}$, the fine electrode patterns were obtained. Using this novel polyimide as an insulating layer, additional fabrication process of wiring is only exposure process without such wet process as development and cleaning. Thus, our surface energy controlled ink-jet printing takes advantage of practical number of process steps.

\section{Results and Discussion}

\subsection{Electrode printing}

Figure 3 shows an optical micrograph of electrode patterns overlaid with the gate insulator (a) without and (b) with the surface energy controlled ink-jet printing. Electrodes without the surface energy controlled ink-jet printing (Figure 3(a)) show droplet-like shape and rough surface with interference fringes, which depends on the film thickness of the gate insulator due to surface roughness of electrodes. On the other hand, electrodes with the surface energy controlled ink-jet printing (Figure $3(b)$ ) show photo mask-like shape and very smooth surface because of no interference fringes.

To investigate the minimum space between two electrodes, we prepared a photo mask with various line and space patterns. After UV irradiation on the novel polyimide film through the photo mask, hydrophilic Ag nanoparticles ink was ink-jetted. Controlling with ink-jet printing conditions such as drop size and ink volume per unit line, two kinds of electrodes with different thicknesses were fabricated. Figure 4 shows the dependence of the yield of electrodes separation on designed space against electrode thickness. Yield means the ratio of separation between the two electrodes and was determined by using an optical microscope with 100 points in one sheet. Figure 4 shows that minimum space up to $2 \mu \mathrm{m}$ (designed) could be fabricated using surface energy controlled ink-jet printing with UV irradiation on the polyimide film. It is superior to conventional ink-jet method.

We also examined alignment margins of electrode fabrication by this ink-jet technique, increasing the distance between the center of high surface energy line pattern and that of ink droplet impinging position every $10 \mu \mathrm{m}$. Figure 5 shows that electrodes with linewidth of $80 \mu \mathrm{m}$ can be successfully formed same as photo mask patterns apart from the center of
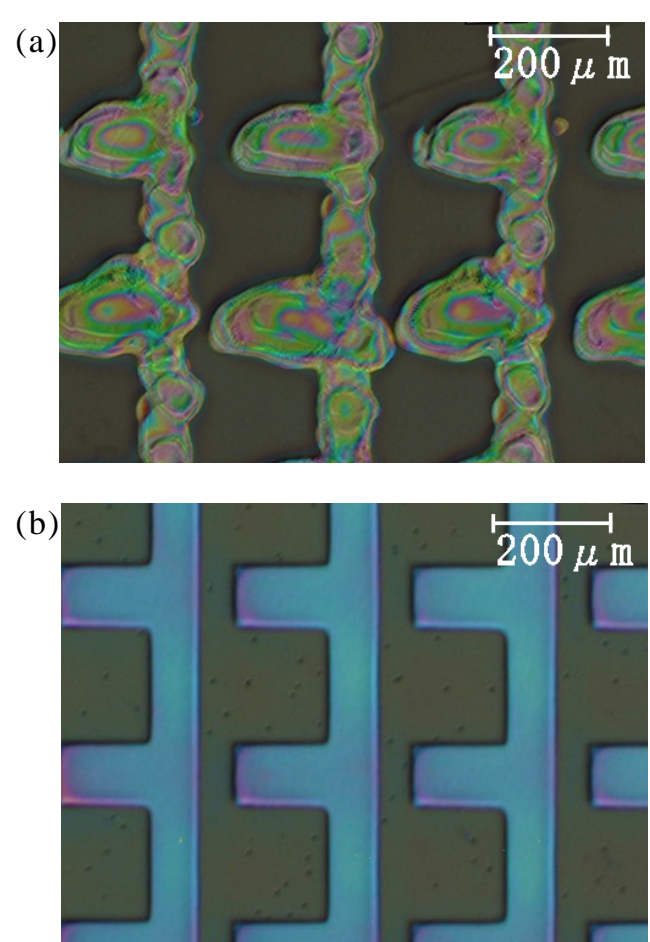

Figure 3. Optical micrograph of electrodes (a) without and (b) with the surface energy controlled ink-jet printing

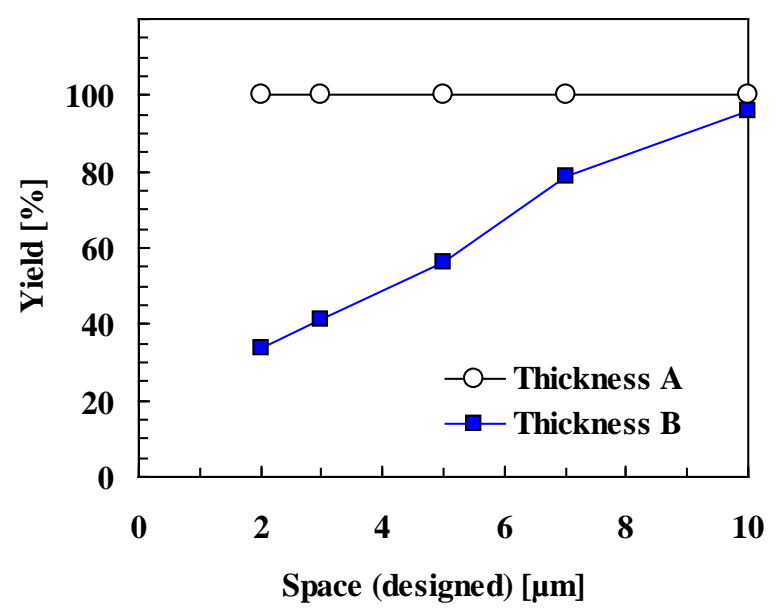

Figure 4. Relationship between yield of electrodes separation and designed space against electrode thickness

the ideal impinging position by a distance of $50 \mu \mathrm{m}$ or less. This is because the hydrophilic ink droplets, which land onto the low surface energy area, could be drawn into the high surface energy area.

Thus, the surface energy controlled ink-jet printing technique possesses such unique features as good surface roughness, high-resolution patterning and high alignment margin because of using a photo mask. 


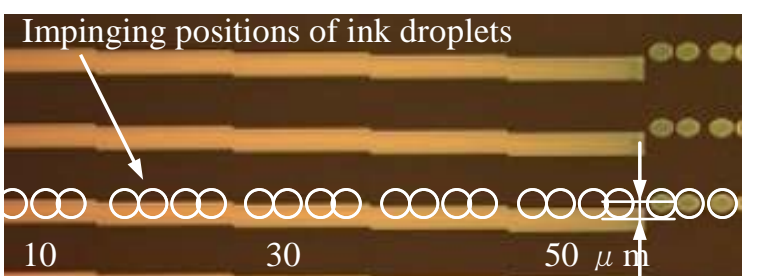

Figure 5. Optical micrograph of electrodes with linewidth of $80 \mu \mathrm{m}$ to show alignment margin of the surface energy controlled ink-jet printing. White circles show impinging position of ink droplets

\subsection{Process integration of TFT array}

For printing process, deposition and pattering is same step. This point of view is very important for process integration of printing process. We fabricated a 160 ppi OTFT backplane with polymer OSC, which consisted of stilbene polymer with triarylamine unit and long-chain alkyl group. Figure 6 shows an optical micrograph of a $160 \mathrm{ppi}$ (pixel size $159 \mu \mathrm{m} \times 159 \mu \mathrm{m}$ ) all-printed OTFT array on flexible substrates after polymer OSC fabrication. Using the appropriate solvent of polymer OSC and optimizing ink-jet conditions, OSC profile was like a coffee stain and separated with each others at $159 \mu \mathrm{m}$ pitches without any bank structure (see Figure 7).

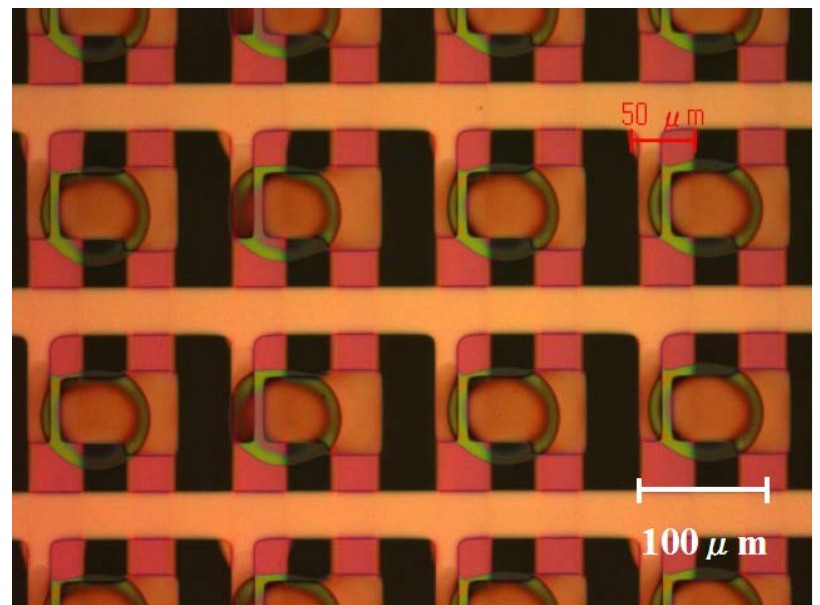

Figure 6. Optical micrograph of a $160 \mathrm{ppi}$ all-printed OTFT backplane on flexible substrate after OSC printing (Channel length $L=5 \mu \mathrm{m}$ )

After OSC printing, insulator and pixel electrode were fabricated by the screen printing.

To shrink the TFT size to 200 ppi (pixel size $127 \mu \mathrm{m} \times 127 \mu \mathrm{m}$ ) from $160 \mathrm{ppi}$ (pixel size 159 $\mu \mathrm{m} \times 159 \mu \mathrm{m})$, we have optimized TFT design such as the line and space of the gate electrode and the S/D electrode. However, it is difficult to achieve fine electrode patterning and low resistivity at same time. Therefore, we optimized

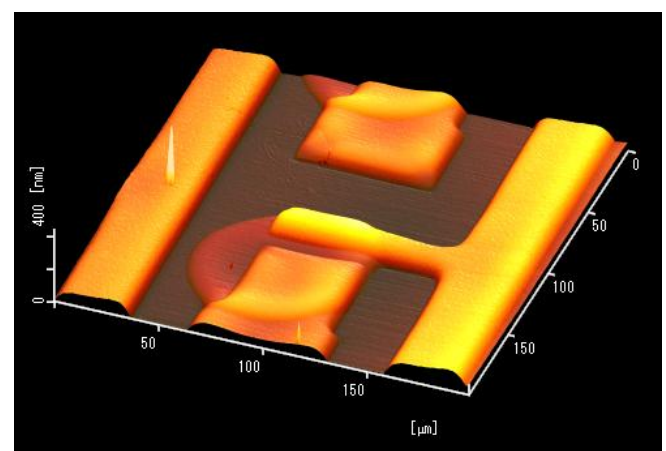

Figure 7. SPM micrograph of a 160 ppi all-printed TFT after OSC printing

the electrode thickness and the curing time for low resistivity. The electrode thickness was controlled by ink-jet condition such as ink volume per unit line.

Figure 8 shows an optical micrograph of a 200 ppi all-printed OTFT array on plastic substrate after $S / D$ electrode fabrication. B oth of the minimum linewidth of the gate line and the source line were $30 \mu \mathrm{m}$. And both of the minimum space of the gate line and the source line were $15 \mu \mathrm{m}$. A minimum width of the source electrode was $15 \mu \mathrm{m}$, channel length was $5 \mu \mathrm{m}$. The resistances per unit length at $30 \mu \mathrm{m}$ linewidth were $1 \mathrm{k} \Omega / \mathrm{cm}$ for the gate line and $0.5 \mathrm{k} \Omega / \mathrm{cm}$ for the source line, respectively, which were enough for 3.2 inch EPDs.

We have also fabricated a 300 ppi (TFT pitch: $85 \mu \mathrm{m} \times 85 \mu \mathrm{m}$ ) all-printed OTFT array on plastic substrate without insulator and pixel electrode (see Figure 9). To shrink the TFT pitch to $85 \mu \mathrm{m}$ from $127 \mu \mathrm{m}$, we designed that the shrinkage of TFT pitch is same as that of source line. Furthermore, we used the OTFT design with the common structure of storage electrode. We optimized surface energy controlled ink-jet printing for electrodes and conventional ink-jet printing for OSC patterning. The minimum channel length $\mathrm{L}$ was up to $3 \mu \mathrm{m}$. The minimum linewidth of the source line was $20 \mu \mathrm{m}$, the minimum space of it was $10 \mu \mathrm{m}$.

\subsection{Performance of OTFT}

Figure 10 shows the transfer characteristics of a 200 ppi all-printed OTFT with $\mathrm{W} / \mathrm{L}=57 \mu \mathrm{m} / 5 \mu \mathrm{m}$ after OSC printing and after pixel electrode printing. Mobility of $0.25 \mathrm{~cm}^{2} / \mathrm{Vs}, \mathrm{V}_{\text {th }}$ of $1.3 \mathrm{~V}$, an on/off current ratio of $10^{6}$ at $\mathrm{V}_{\mathrm{ds}}=-20 \mathrm{~V}$ were obtained after small- 


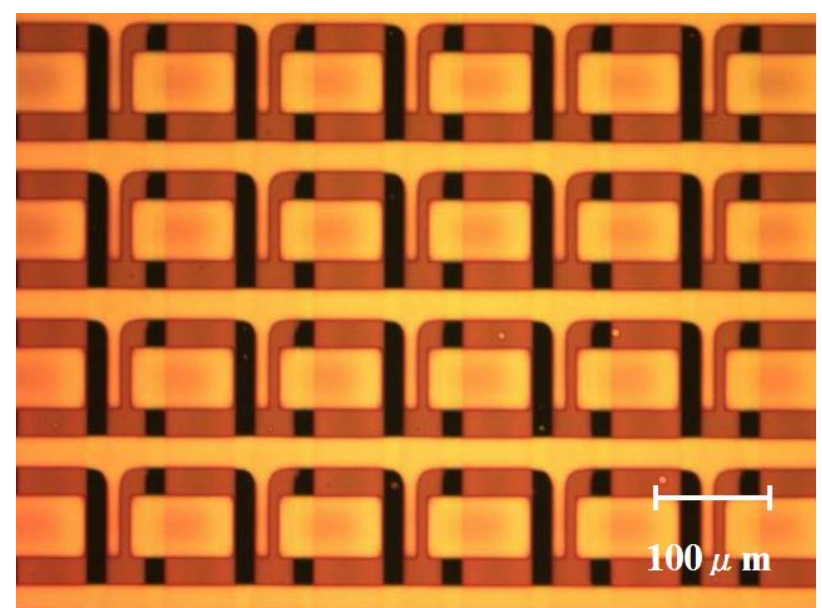

Figure 8. Optical micrograph of a $200 \mathrm{ppi}$ all-printed OTFT backplane on flexible substrate after $S / D$ electrode printing ( $C$ hannel length $L=5$ $\mu \mathrm{m})$

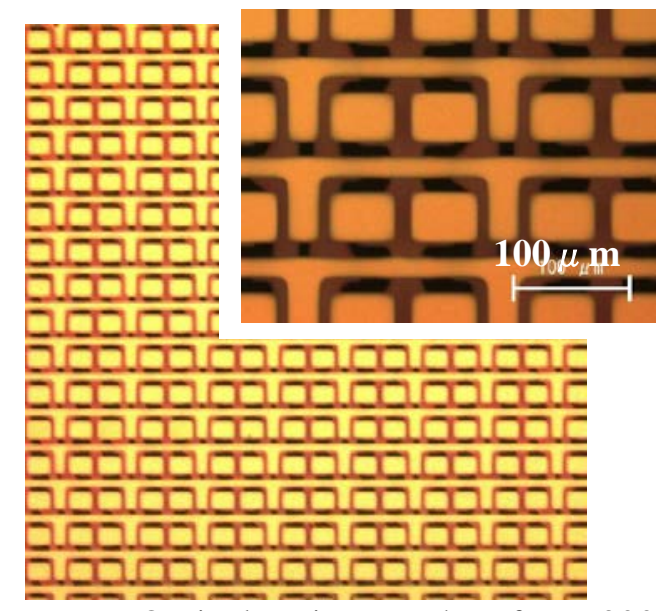

Figure 9. Optical micrograph of a $300 \mathrm{ppi}$ all-printed OTFT array on flexible substrate after $\mathrm{S} / \mathrm{D}$ electrode printing (Channel length $\mathrm{L}=5 \mu \mathrm{m}$ )

molecule OSC printing. High on/off current ratio is because of the separation of

small-molecule OSC patterning with high mobility of TFT. We optimized the surface of the gate insulator and OSC ink formulation such as the solvent and the concentration. Although a slight reduction in the on-current and a small $\mathrm{V}_{\text {th }}$ shift were observed after pixel electrode printing, mobility of $0.12 \mathrm{~cm}^{2} / \mathrm{V} \mathrm{s}$, $V_{\text {th }}$ of $3.9 \mathrm{~V}$ were still maintained, which is sufficient for driving high resolution EPDs.

Figure 11 shows the transfer characteristics of a 300 ppi all-printed OTFT with $\mathrm{W} / \mathrm{L}=45 \mu \mathrm{m} / 5 \mu \mathrm{m}$ after OSC printing. M obility of $0.32 \mathrm{~cm}^{2} / \mathrm{V} \mathrm{s}, V$ th of $1.5 \mathrm{~V}$, an on/off current ratio of $10^{6}$ at $\mathrm{V}$ ds $=-20$ $\mathrm{V}$ were obtained after small-molecule OSC printing. These value were almost same as those of 200 ppi all-printed OTFT.

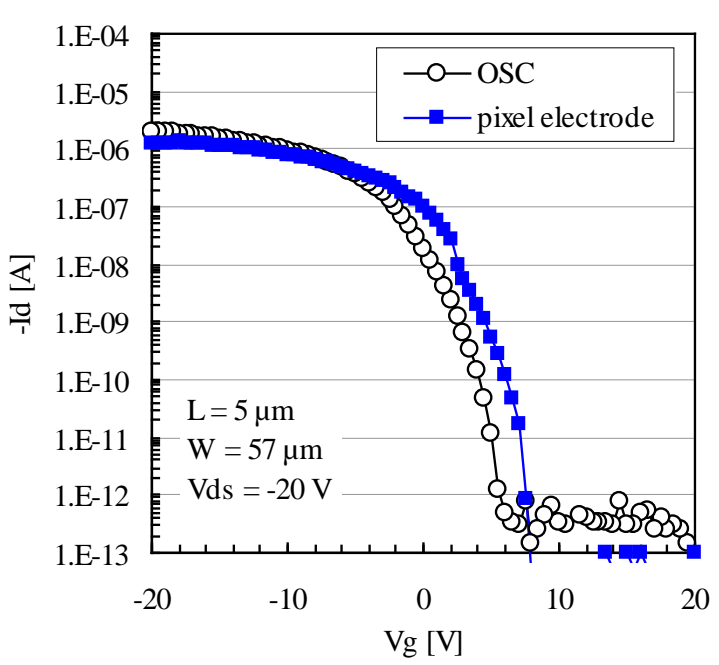

Figure 10. Transfer characteristics of a $200 \mathrm{ppi}$ all-printed OTFT after OSC printing and after pixel electrode printing ( $C$ hannel length $L=5$ $\mu \mathrm{m})$

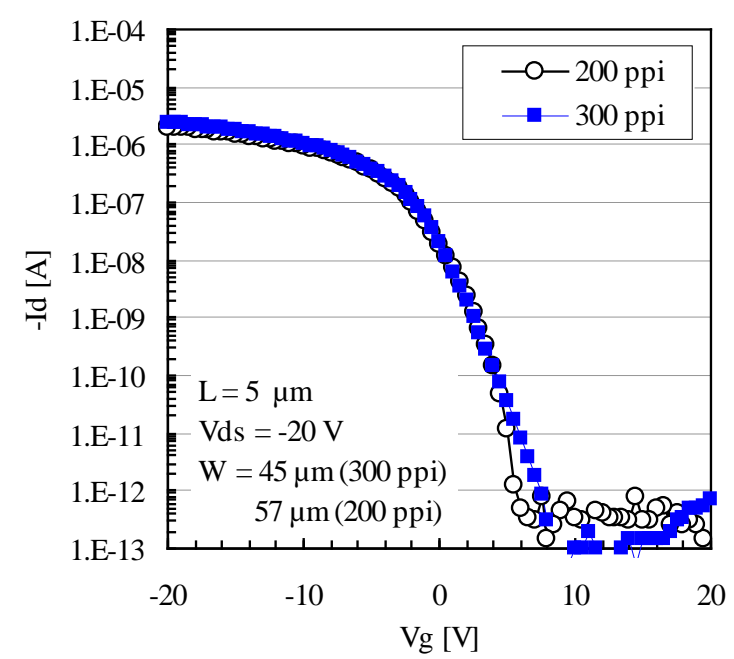

Figure 11. Transfer characteristics of a $300 \mathrm{ppi}$ all-printed OTFT after OSC printing (Channel length $\mathrm{L}=5 \mu \mathrm{m}$ )

\subsection{Demonstration of Flexible electric paper}

For demonstration of flexible EPDs, electrophoretic sheets were laminated with a 200 ppi all-printed OTFT backplanes on plastic substrate (see Figure 12). Japanese characters with 6-point Ming-style font were successfully displayed with bended EPDs driven by a 200 ppi all-printed OTFT backplane (see Figure 13). The applied voltages of select line and data line were 30 $\mathrm{V} p \mathrm{p}$ and $20 \mathrm{Vpp}$, respectively. 


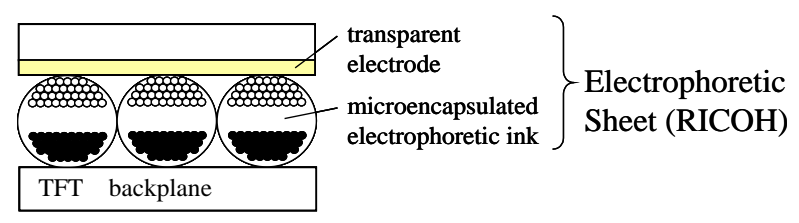

Figure 12. Schematic structure of flexible EPDs.

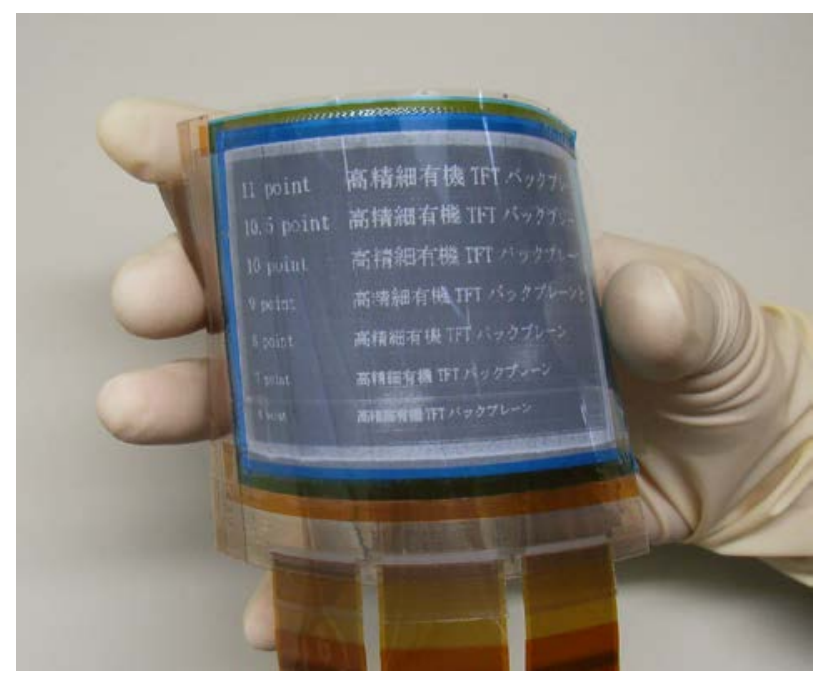

Figure 13. Photograph of a 3.2 inch flexible EPD driven by a $200 \mathrm{ppi}$ all-printed OTFT backplane.

\section{Conclusion}

We have developed the surface energy controlled ink-jet printing with UV irradiation for A g electrodes and the conventional ink-jet printing for OSC. To integrate these printing methods we have succeeded in fabricating 160, 200 ppi all-printed OTFT backplanes and 300 ppi all-printed OTFT array on plastic substrates. Flexible electric paper driven by 200 ppi all-printed OTFT backplanes was also successfully demonstrated. The resolution of $200 \mathrm{ppi}$ and mobility of over $0.1 \mathrm{~cm}^{2} / \mathrm{V} \mathrm{s}$ are enough for a monochrome electronic paper. This printing technology is promising for high-resolution, low-cost and low-environmental impact manufacturing process.

\section{R eferences}

1. Z. B ao, Y. Feng, A. Dodabalapur, V. R. Raju, and A. J. Lovinger, Chem. Mater., 9 (1997), 1299.

2. H. Sirringhause, T. Kawase, R. H. Friend, T. Shimoda, M. Inbasekaran, W. Wu, and E. P. Woo, Science, 290 (2000), 2123.

3. A. C. A rias, J. Daniel, B. K rusor, S. Ready,
V. Sholin, and R. Street, Journal of the SID, Vol. 15, No. 7 (2007), 485.

4. V. Subramanian, J. Frechet, P. Chang, D. C. Huang, J. Lee, S. Molesa, A. M urphy, D. Redinger, and $\mathrm{S}$. Vilkman, Proceeding of the IEEE, Vol.93, No.7 (2005), 1330.

5. Y. Noguchi, T. Sekitani, and T. Someya, Appl. Phys. Lett., 89 (2006), 253507.

6. T. A rai, N. Sato, K. Yamaguchi, M. Kawasaki, M. Fujimori, T. Shiba, M. Ando, and K. Torii, Jpn. J. Appl. Phys., 46(4B) (2007), 2700.

7. T. Okubo, Y. Kokubo, K. Hatta, R. Matsubara, M. Ishizaki, Y. Ugajin, N. Sekine, N. K awashima, T. Fukuda, A. Nomoto, T. Ohe, N. Kobayashi, K. Nomoto, and J. Kasahara, Proc. IDW'07, (2007), 463.

8. Y. Ito, R. Matsubara, O. Kina, $M$. Ishizaki and M. Ito, Proc. IDW'10, (2010), 467

9. H. Maeda, H. Honda, M. Matsuoka, M. Nagae, T. Suzuki, K. Ogawa, and $H$. K obayashi, Proc. ID W08, (2008), 1469.

10. K. M atsuoka, O. Kina, M. Koutake, K. Noda, H. Yonehara, K. Nakanishi, and K. Yase, Proc. IDW'09, (2009), 717.

11. N. Kawashima, N. K obayashi, N. Yoneya, H. Ono, T. Fukuda, T. Ohe, Y. Ishii, A. Nomoto, M. Sasaki, and K. Nomoto, Proc. SID'09, (2009), 25.

12. T. Tano, H. Tomono, H. Kondoh, and K. Fujimura, Proc. AMLCD2004, (2004), 37.

13.K. Suzuki, K. Yutani, A. Onodera, T. Tano, H. Tomono, A. M urakami, M. Yanagisawa, K. K ameyama, and I. Kawashima, Proc. IDW'08 (2008), 1477.

14. K. Suzuki, K. Yutani, M. Nakashima, A. Onodera, S. M izukami, M. K ato, T. Tano, $H$. Tomono, $M$. Yanagisawa, and $K$. K ameyama, Proc. ID W'09, (2009), 1581.

15. K. Suzuki, K. Yutani, M. Nakashima, A. Onodera, S. M izukami, M. K ato, T. Tano, $\mathrm{H}$. Tomono, M. Yanagisawa, and $\mathrm{K}$. K ameyama, Proc. ISE P 2010, (2010).

16. T. Yamaga, T. Sagisaka, and Y. A kiyama, Abstracts of Papers, MRS $2007 \mathrm{~F}$ all M eeting, (2007), G1.10. 